\title{
Abdominal ectopic pregnancy after in vitro fertilization and single embryo transfer: a case report and systematic review
}

\author{
Nicole Yoder, Reshef Tal ${ }^{*}$ (D) and J. Ryan Martin
}

\begin{abstract}
Background: Ectopic pregnancy is the leading cause of maternal morbidity and mortality during the first trimester and the incidence increases dramatically with assisted-reproductive technology (ART), occurring in approximately 1. $5-2.1 \%$ of patients undergoing in-vitro fertilization (IVF). Abdominal ectopic pregnancy is a rare yet clinically significant form of ectopic pregnancy due to potentially high maternal morbidity. While risk factors for ectopic pregnancy after IVF have been studied, very little is known about risk factors specific for abdominal ectopic pregnancy. We present a case of a 30 year-old woman who had an abdominal ectopic pregnancy following IVF and elective single embryo transfer, which was diagnosed and managed by laparoscopy. We performed a systematic literature search to identify case reports of abdominal or heterotopic abdominal ectopic pregnancies after IVF. A total of 28 cases were identified.

Results: Patients' ages ranged from 23 to 38 (Mean 33.2, S.D. = 3.2). Infertility causes included tubal factor (46 \%), endometriosis (14\%), male factor (14\%), pelvic adhesive disease (7\%), structural/DES exposure (7\%), and unexplained infertility (14\%). A history of ectopic pregnancy was identified in $39 \%$ of cases. A history of tubal surgery was identified in $50 \%$ of cases, $32 \%$ cases having had bilateral salpingectomy. Transfer of two embryos or more (79 \%) and fresh embryo transfer (71\%) were reported in the majority of cases. Heterotopic abdominal pregnancy occurred in $46 \%$ of cases while $54 \%$ were abdominal ectopic pregnancies.

Conclusions: Our systematic review has revealed several trends in reported cases of abdominal ectopic pregnancy after IVF including tubal factor infertility, history of tubal ectopic and tubal surgery, higher number of embryos transferred, and fresh embryo transfers. These are consistent with known risk factors for ectopic pregnancy following IVF. Further research focusing on more homogenous population may help in better characterizing this rare IVF complication and its risks.
\end{abstract}

Keywords: Abdominal pregnancy, Ectopic pregnancy, In vitro fertilization, IVF-ET

\section{Background}

Ectopic pregnancy is the leading cause of maternal morbidity and mortality during the first trimester and the incidence increases dramatically with assisted reproductive technology (ART), occurring in approximately $1.5-2.1 \%$ of patients undergoing IVF $[1,2]$. The majority of ectopic pregnancies from either IVF or spontaneous pregnancy occur within the fallopian tubes, but implantation may occur in other locations such as the cervix, ovary,

\footnotetext{
*Correspondence: reshef.tal@yale.edu

Division of Reproductive Endocrinology \& Infertility, Department of

Obstetrics, Gynecology, \& Reproductive Sciences, Yale University School of

Medicine, 333 Cedar Street, New Haven, CT 06510, USA
}

or abdomen [3]. Abdominal ectopic pregnancies are a very rare form of ectopic pregnancy, yet are clinically significant due to their potential for high morbidity and often atypical presentation [4].

Recent studies have attempted to identify risk factors for ectopic pregnancy after IVF. Suggested risk factors include infertility due to tubal factor, endometriosis, transfer at blastocyst stage, higher number of embryos transferred, decreased endometrial thickness, variation in culture media, and fresh embryo transfer [5-9]. However, very little data exists regarding risk factors for abdominal ectopic pregnancy after IVF. 
In this case study, we report an abdominal ectopic pregnancy after IVF with fresh single embryo transfer. We also performed a systematic review of the literature for known cases of abdominal ectopic pregnancy after IVF and provide detailed characterization of these patients and risk factors for this rare complication.

\section{Case description}

The patient was a 30-year-old G2P0010 who presented to our fertility center seeking fertility treatment. She had a medical history of polycystic ovarian syndrome (PCOS) and her partner had a diagnosis of male factor infertility. She had no prior surgical history, no known allergies, and medications included prenatal vitamins. She denied any history of sexually transmitted infections and had a normal hysterosalpingogram and saline sonohysterogram. Her first IVF cycle with an elective single embryo transfer resulted in a negative pregnancy test. Her second IVF cycle used a GnRH antagonist stimulation protocol and she was triggered with Ovidrel on stimulation day 12. Twenty-two oocytes were retrieved. On day five a single fresh blastocyst was transferred using a pass through technique under ultrasound guidance. A stiff outer sheath was introduced through the cervix and past the internal os. A soft tipped catheter containing the embryo was advanced through the outer sheath and the embryo was expelled into the uterine cavity approximately $1.5 \mathrm{~cm}$ from the uterine fundus with good visualization. Beta hCG was positive on post-transfer day 9 and serial beta hCG values were monitored and continued to rise appropriately (Table 1 ). On day 28 after embryo transfer, the patient underwent a transvaginal ultrasound (TVUS) in the office that did not identify an intrauterine pregnancy (IUP) or any abnormal adnexal structures. She was asymptomatic with no vaginal

Table 1 Beta hCG level and timeline of events

\begin{tabular}{lll}
\hline Day & Beta HCG pg/mL & Event \\
\hline-5 & & Oocyte retrieval, ICSI \\
0 & & Day 5 single embryo transfer \\
9 & 28.7 & \\
11 & 45.5 & \\
13 & 130 & \\
15 & 382 & \\
17 & 991 & \\
19 & 2020 & Sac Check - No IUP or adnexal abnormalities \\
28 & 12,400 & Methotrexate given \\
29 & 13,000 & \\
32 & 20,000 & TVUS - Right adnexal mass with gestational sac \\
33 & & and fetal cardiac activity \\
34 & & Diagnostic laparoscopy - Abdominal ectopic \\
\hline
\end{tabular}

bleeding or abdominal pain. The patient was sent for a more comprehensive ultrasound evaluation at the associated Maternal Fetal Medicine unit and another beta hCG value was obtained. Repeat scan similarly failed to identify an IUP or visualize an ectopic pregnancy. The beta hCG was $12,400 \mathrm{pg} / \mathrm{mL}$. Given the high beta hCG value in the absence of an IUP, the patient was counseled and advised to take methotrexate treatment for presumed ectopic pregnancy of unknown location. One day later (day 29), she received an intramuscular dose of $83 \mathrm{mg}\left(50 \mathrm{mg} / \mathrm{m}^{2}\right.$ body surface area) methotrexate with plans to follow up with repeat beta hCG and TVUS.

Four days after methotrexate administration, repeat beta hCG level continued to rise $(20,000 \mathrm{pg} / \mathrm{mL})$ and an ultrasound performed 1 day later demonstrated a right adnexal mass with a yolk sac, fetal pole, and fetal cardiac activity. The decision was made to proceed with diagnostic laparoscopy for treatment of ectopic pregnancy after failure of methotrexate therapy. The patient continued to be asymptomatic with no vaginal bleeding or abdominal pain. Diagnostic laparoscopy was performed on day 34 post-embryo transfer. The operative findings were significant for minimal hemoperitoneum $(<50 \mathrm{~mL})$ and products of conception were noted to be implanted on the peritoneum of the posterior cul-de-sac medial to the left uterosacral ligament (Fig. 1). The products of conception were removed using graspers without difficulty and hemostasis was obtained with electrocautery and surgicel. All other pelvic organs including uterus and bilateral ovaries and tubes appeared grossly normal in appearance.

\section{Systematic review of the literature}

A systematic literature review was performed with the aim of identifying all other case reports of abdominal ectopic pregnancies after IVF. The literature search was performed using PubMed, Google Scholar, and EMBASE without language restriction encompassing publications until July 2016. Search terms used included 'IVF', 'ectopic pregnancy', 'abdominal ectopic pregnancy', and 'heterotopic pregnancy'. To the best of our knowledge, all reported cases and available data are summarized in Table 2.

\section{Results}

A total of 28 cases of abdominal ectopic pregnancy after IVF were identified. The age of patients ranged from 23 to 38 yo $($ Mean $=33.2$ S.D. $=3.2$ ), with no age reported in 1 case. Infertility causes included tubal factor in 13 (46\%) cases, endometriosis in $4(14 \%)$ cases, male factor in $4(14 \%)$ cases, pelvic adhesive disease in $2(7 \%)$ cases, structural/DES exposure in $2(7 \%)$ cases, unexplained in $4(14 \%)$ cases, and one case did not specify the cause. Overall, anatomic/structural factors accounted 


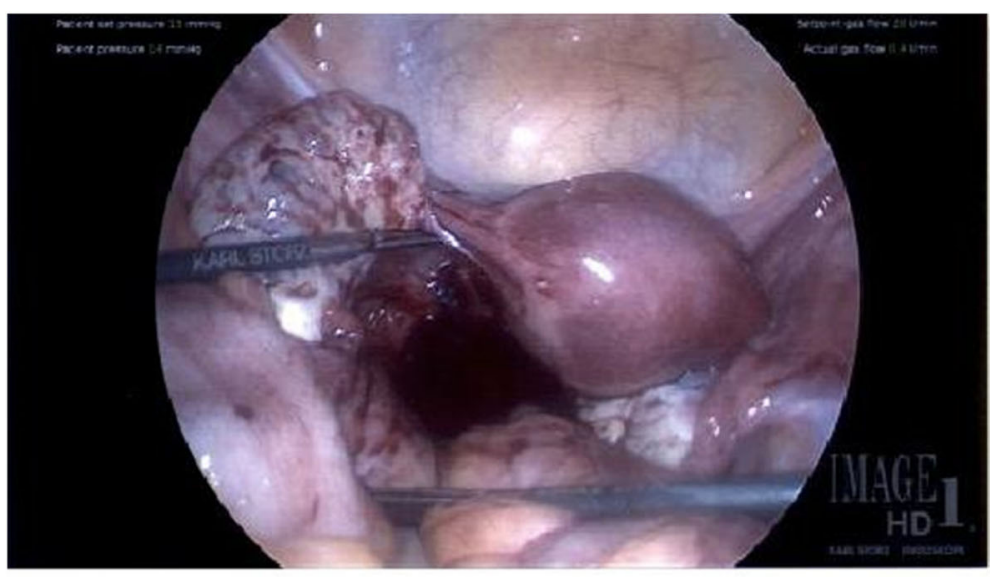

IMG002

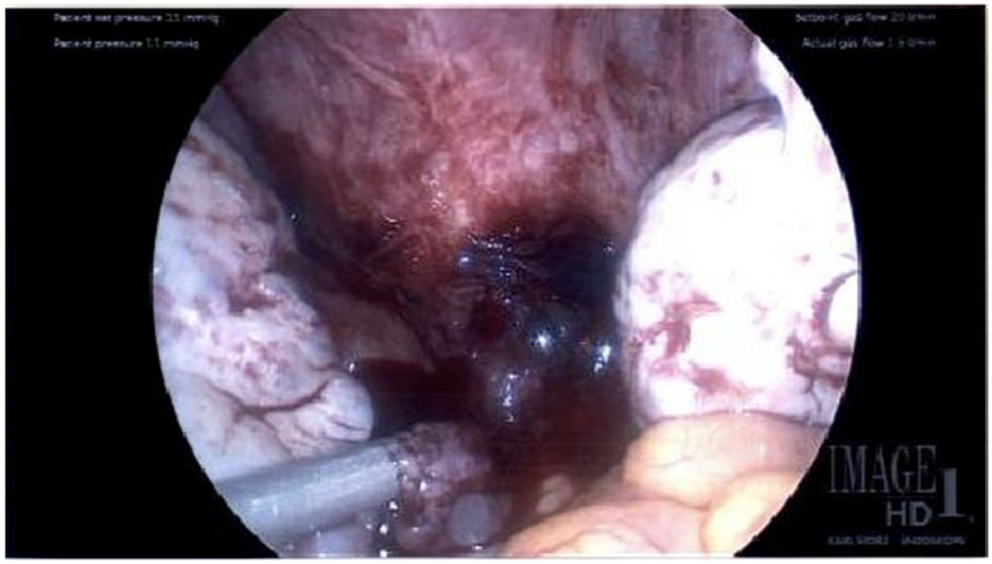

Fig. 1 Diagnostic laparoscopy demonstrating hemoperitoneum (top image) and products of conception implanted in the posterior cul-de-sac (bottom image)

for 17 (61 \%) of the cases. A history of ectopic pregnancy was identified in 11 (39\%) cases. History of tubal surgery had been described in 14 (50 \%) cases, 9 (32\%) of which were bilateral salpingectomy. Transfer of more than two embryos was reported in 15 (54 \%) cases, two embryos were transferred in 7 (25\%) cases, while single embryo transfer was reported in only two (7\%) cases. No information about number of embryos transferred was available in $4(14 \%)$ cases. Fresh embryo transfer accounted for $20(71 \%)$ cases, frozen embryo transfer in $3(11 \%)$ cases, and $5(18 \%)$ cases did not specify fresh versus frozen embryo transfer. Heterotopic abdominal pregnancy occurred in 13 (46\%) cases, and 15 (54\%) were abdominal ectopic pregnancies. Notable cases include 5 retroperitoneal ectopic pregnancies, an abdominal fetal demise at 28 weeks, and 4 cases of viable abdominal pregnancies at 30 weeks, 32 weeks (two cases), and 34 weeks gestation.

\section{Discussion}

Abdominal ectopic pregnancies comprise less than $1 \%$ of all ectopic pregnancies, yet have a maternal mortality rate eight times greater than tubal ectopic pregnancies [10]. For this reason, early recognition and treatment is crucial in the setting of abdominal ectopic pregnancy. The case presented demonstrates the diagnostic challenge of abdominal ectopic, as the patient's beta hCG values followed a normal rise and the patient remained asymptomatic up to the point of diagnostic laparoscopy. Transvaginal ultrasound did not visualize the ectopic pregnancy until the beta $\mathrm{hCG}$ value was $20,000 \mathrm{pg} / \mathrm{mL}$, which is far beyond the usual discriminatory zone. This atypical presentation of an ectopic pregnancy highlights the need to consider abdominal ectopic pregnancy in the differential of any pregnancy of unknown location after IVF, especially in the setting of non-diagnostic transvaginal ultrasound.

There appears to be an increased rate of ectopic pregnancies after ART when compared to rates in spontaneous pregnancy [11]. As the number of IVF procedures performed continues to rise, the incidence of ectopic and abdominal ectopic pregnancy will likely also rise. While there are still relatively few reported cases of abdominal ectopic pregnancies after IVF, our systematic 
Table 2 Abdominal ectopic case reports

\begin{tabular}{|c|c|c|c|c|c|c|c|c|c|c|c|c|c|c|}
\hline $\begin{array}{l}\text { Author } \\
\text { (year) }\end{array}$ & $\begin{array}{l}\text { Age/ } \\
\text { Parity }\end{array}$ & $\begin{array}{l}\text { Infertility } \\
\text { etiology }\end{array}$ & $\begin{array}{l}\text { Other pertinent } \\
\text { history }\end{array}$ & Priorectopic & $\begin{array}{l}\text { Stimulation } \\
\text { Protocol }\end{array}$ & $\begin{array}{l}\text { Egg } \\
\#\end{array}$ & $\begin{array}{l}\text { ET } \\
\text { no./ } \\
\text { timing }\end{array}$ & $\begin{array}{l}\text { Fresh/ } \\
\text { Frozen } \\
\text { ET }\end{array}$ & $\begin{array}{l}\text { Max } \\
\text { HCG } \\
\text { level } \\
(\mathrm{mlU} / \\
\mathrm{ml})\end{array}$ & Location $(E / H)$ & $\begin{array}{l}\text { Stage at } \\
\text { diagnosis }\end{array}$ & Rupture? & Intervention & Outcome \\
\hline $\begin{array}{l}\text { Oehniger } \\
\text { (1988) } \\
{[23]}\end{array}$ & $\begin{array}{l}35 \text { yo } \\
\text { GOPO }\end{array}$ & Endometriosis & $\begin{array}{l}\text { Laparotomy x } \\
\text { 2, left } \\
\text { salpingectomy, } \\
\text { frozen pelvis; } \\
\text { Right } \\
\text { hydrosalpinx } \\
\text { with partial } \\
\text { obstruction }\end{array}$ & No & $\begin{array}{l}\text { FSH/Pergonal } \\
\text { (hMG/hCG), } \\
\text { hCG trigger }\end{array}$ & 4 & $\begin{array}{l}4 \\
42- \\
44 \mathrm{~h}\end{array}$ & Fresh & NA & $\begin{array}{l}\text { Sigmoid } \\
\text { mesentery (E) }\end{array}$ & $\sim 41$ days PT & No & $\begin{array}{l}\text { Exploratory } \\
\text { Laparotomy }\end{array}$ & $\begin{array}{l}\text { Removal of } \\
\text { pregnancy } \\
\text { tissue by } \\
\text { laparotomy }\end{array}$ \\
\hline $\begin{array}{l}\text { Bassil } \\
\text { (1991) } \\
\text { [24] }\end{array}$ & $\begin{array}{l}33 \text { yo } \\
\mathrm{NA}\end{array}$ & Male factor & NA & NA & $\begin{array}{l}\text { Clomid/hMG, } \\
\text { hCG trigger }\end{array}$ & 6 & $\begin{array}{l}4 \\
\text { NA }\end{array}$ & Fresh & NA & $\begin{array}{l}\text { Posterior uterus, } \\
\text { broad ligament } \\
(\mathrm{H})\end{array}$ & $\begin{array}{l}19 \text { weeks } \\
\text { gestation }\end{array}$ & No & $\begin{array}{l}\text { Laparotomy, } \\
\text { right } \\
\text { adnexectomy }\end{array}$ & $\begin{array}{l}\text { Delivery of } \\
\text { viable twins } \\
\text { at } 34 \text { weeks }\end{array}$ \\
\hline $\begin{array}{l}\text { Ferland } \\
(1991) \\
{[25]}\end{array}$ & $\begin{array}{l}32 \text { yo } \\
\text { G4P0030 }\end{array}$ & $\begin{array}{l}\text { DES } \\
\text { exposure, } \\
\text { secondary } \\
\text { infertility }\end{array}$ & $\begin{array}{l}\text { Right } \\
\text { salpingectomy, } \\
\text { left hydrosalpinx }\end{array}$ & $\begin{array}{l}\text { Tubal } \\
\text { ectopic }\end{array}$ & $\begin{array}{l}\text { Long } \\
\text { protocol w/ } \\
\text { GnRH agonist }\end{array}$ & 7 & $\begin{array}{l}3 \\
\text { Day } \\
2 \mathrm{ET}\end{array}$ & Fresh & 19,450 & $\begin{array}{l}\text { Retroperitoneal } \\
\text { (E) }\end{array}$ & 37 days PT & Yes & $\begin{array}{l}\text { Laparotomy, left } \\
\text { salpingectomy }\end{array}$ & \\
\hline $\begin{array}{l}\text { Ragni } \\
\text { (1991) } \\
\text { [26] }\end{array}$ & $\begin{array}{l}32 \text { yo } \\
\text { G1P0010 }\end{array}$ & $\begin{array}{l}\text { Pelvic } \\
\text { adhesive } \\
\text { disease }\end{array}$ & $\begin{array}{l}\text { Right } \\
\text { adnexectomy, } \\
\text { hysteropexy }\end{array}$ & $\begin{array}{l}\text { Tubal } \\
\text { ectopic }\end{array}$ & $\begin{array}{l}\text { Long } \\
\text { protocol w/ } \\
\text { GnRH agonist }\end{array}$ & 4 & $\begin{array}{l}3 \\
\text { Day } \\
2 \mathrm{ET}\end{array}$ & Fresh & NA & $\begin{array}{l}\text { Right adnexa } \\
(\mathrm{H})\end{array}$ & $\begin{array}{l}12 \text { weeks } \\
\text { gestation }\end{array}$ & No & $\begin{array}{l}\text { Selective } \\
\text { reduction of } \\
\text { abdominal } \\
\text { pregnancy, } \\
\text { laparotomy }\end{array}$ & $\begin{array}{l}\text { Laparotomy } \\
\text { for resorbing } \\
\text { abdominal } \\
\text { pregnancy, } \\
\text { SAB of IUP at } \\
16 \text { weeks }\end{array}$ \\
\hline $\begin{array}{l}\text { Balmaceda } \\
\text { (1993) [27] }\end{array}$ & $\begin{array}{l}33 \text { yo } \\
\text { G3P1021 }\end{array}$ & Tubal & $\begin{array}{l}\text { Right } \\
\text { salpingectomy, } \\
\text { left } \\
\text { salpingostomy }\end{array}$ & $\begin{array}{l}\text { Tubal } \\
\text { Ectopic } \times 2\end{array}$ & $\begin{array}{l}\text { Short } \\
\text { protocol, w/ } \\
\text { GnRH agonist }\end{array}$ & 15 & $\begin{array}{l}4 \\
\text { Day } \\
4 \mathrm{ET}\end{array}$ & Fresh & 4651 & $\begin{array}{l}\text { Abdominal - } \\
\text { broad ligament } \\
\text { (E) }\end{array}$ & 30 days PT & No & $\begin{array}{l}\text { Laparoscopy, } \\
\text { salpingectomy }\end{array}$ & $\begin{array}{l}\text { Laparoscopic } \\
\text { removal of } \\
\text { abdominal } \\
\text { ectopic, left } \\
\text { salpingectomy }\end{array}$ \\
\hline $\begin{array}{l}\text { Fisch } \\
\text { (1995) } \\
{[28]}\end{array}$ & $\begin{array}{l}32 \text { yo } \\
\text { G2P0020 }\end{array}$ & Tubal & $\begin{array}{l}\text { Bilateral } \\
\text { salpingectomy }\end{array}$ & $\begin{array}{l}\text { Tubal } \\
\text { ectopic } \times 2\end{array}$ & $\begin{array}{l}\text { Long } \\
\text { protocol w/ } \\
\text { GnRH agonist }\end{array}$ & 5 & $\begin{array}{l}3 \\
\text { NA }\end{array}$ & Fresh & NA & $\begin{array}{l}\text { lleum, left } \\
\text { uterine cornua } \\
(\mathrm{H})\end{array}$ & $\begin{array}{l}10 \text { weeks } \\
\text { gestation }\end{array}$ & Yes & $\begin{array}{l}\text { Gastrostoscopy, } \\
\text { sigmoidoscopy, } \\
\text { Tc scan, } \\
\text { angiography, } \\
\text { D\&C, tagged } \\
\text { RBC scan, } \\
\text { Laparotomy }\end{array}$ & $\begin{array}{l}\text { Laparotomy } \\
\text { for abdominal } \\
\text { ectopic, D\&C } \\
\text { for incomplete } \\
\text { AB of IUP }\end{array}$ \\
\hline $\begin{array}{l}\text { DelRosario } \\
\text { (1996) [29] }\end{array}$ & $\begin{array}{l}33 \text { yo } \\
\text { G1P1001 }\end{array}$ & Tubal & Breast Cancer & No & NA & NA & $\begin{array}{l}4 \\
\text { NA }\end{array}$ & Frozen & 563 & Bladder (E) & 75 days PT & Yes & $\begin{array}{l}\text { Methotrexate, } \\
\text { laparoscopy }\end{array}$ & $\begin{array}{l}\text { Laparoscopic } \\
\text { removal of } \\
\text { pregnancy } \\
\text { tissue }\end{array}$ \\
\hline
\end{tabular}


Table 2 Abdominal ectopic case reports (Continued)

\begin{tabular}{|c|c|c|c|c|c|c|c|c|c|c|c|c|c|c|}
\hline $\begin{array}{l}\text { Fisch } \\
\text { (1996) } \\
{[11]}\end{array}$ & $\begin{array}{l}38 \text { yo } \\
\text { G2P0020 }\end{array}$ & Tubal & $\begin{array}{l}\text { Laparoscopic } \\
\text { Salpingectomy } x 2 \text {, } \\
\text { 8th IVF cycle }\end{array}$ & $\begin{array}{l}\text { Tubal } \\
\text { ectopic } \times 2\end{array}$ & $\begin{array}{l}\text { Long } \\
\text { protocol w/ } \\
\text { GnRH agonist }\end{array}$ & 14 & $\begin{array}{l}4 \\
\text { Day } \\
3 \mathrm{ET}\end{array}$ & Fresh & 1730 & $\begin{array}{l}\text { Broad Ligament } \\
\text { (E) }\end{array}$ & 21 days PT & Yes & $\begin{array}{l}\text { Exploratory } \\
\text { Laparotomy }\end{array}$ & $\begin{array}{l}\text { Removal of } \\
\text { pregnancy } \\
\text { tissue by } \\
\text { laparotomy }\end{array}$ \\
\hline $\begin{array}{l}\text { Moonen- } \\
\text { Delarue } \\
(1996) \\
{[30]}\end{array}$ & $\begin{array}{l}23 \text { yo } \\
\text { G2P0020 }\end{array}$ & $\begin{array}{l}\text { Pelvic } \\
\text { adhesive } \\
\text { disease }\end{array}$ & $\begin{array}{l}\text { Right } \\
\text { salpingectomy }\end{array}$ & $\begin{array}{l}\text { Tubal and } \\
\text { abdominal } \\
\text { ectopic }\end{array}$ & NA & NA & $\begin{array}{l}\text { NA } \\
\text { NA }\end{array}$ & Fresh & NA & $\begin{array}{l}\text { Abdominal - } \\
\text { uterine fundus } \\
\text { (E) }\end{array}$ & 28 weeks & $\begin{array}{l}\text { Placental } \\
\text { abruption }\end{array}$ & Laparotomy & $\begin{array}{l}\text { Fetal demise } \\
\text { of abdominal } \\
\text { ectopic @ } \\
28 \text { weeks }\end{array}$ \\
\hline $\begin{array}{l}\text { Pisarska } \\
\text { (1998) } \\
{[31]}\end{array}$ & $\begin{array}{l}35 \text { yo } \\
\text { G2P0020 }\end{array}$ & Unexplained & NA & No & $\begin{array}{l}\text { Long } \\
\text { protocol w/ } \\
\text { GnRH agonist }\end{array}$ & 9 & $\begin{array}{l}6 \\
\text { NA }\end{array}$ & Fresh & 6004 & $\begin{array}{l}\text { Bladder serosa } \\
(\mathrm{H})\end{array}$ & $\begin{array}{l}6 \text { weeks } \\
\text { gestation }\end{array}$ & No & $\begin{array}{l}\text { Diagnostic } \\
\text { laparoscopy }\end{array}$ & $\begin{array}{l}\text { Laparoscopic } \\
\text { removal of } \\
\text { ectopic } \\
\text { pregnancy } \\
\text { (bladder), term } \\
\text { delivery of IUP }\end{array}$ \\
\hline $\begin{array}{l}\text { Deshpande } \\
\text { (1999) [32] }\end{array}$ & $\begin{array}{l}33 \text { yo } \\
\text { G1P0010 }\end{array}$ & Endometriosis & $\begin{array}{l}\text { Endometriosis, } \\
\text { left } \\
\text { salpingectomy, } \\
\text { Patent right } \\
\text { tube }\end{array}$ & No & $\begin{array}{l}\text { Long } \\
\text { protocol w/ } \\
\text { GNRH } \\
\text { agonist }\end{array}$ & 8 & $\begin{array}{l}2 \\
\text { Day } \\
3 \mathrm{ET}\end{array}$ & Fresh & 55,560 & $\begin{array}{l}\text { Twin pregnancy } \\
\text { in broad } \\
\text { ligament }(\mathrm{H})\end{array}$ & 7 weeks PT & No & Laparotomy & $\begin{array}{l}\text { Removal of } \\
\text { twin ectopic } \\
\text { pregnancy by } \\
\text { laparotomy at } \\
7 \text { weeks }\end{array}$ \\
\hline $\begin{array}{l}\text { Scheiber } \\
\text { (1999) } \\
\text { [33] }\end{array}$ & $\begin{array}{l}37 \text { yo } \\
\text { G3P0030 }\end{array}$ & $\begin{array}{l}\text { Tubal factor } \\
\text { EndometriosisDOR }\end{array}$ & $\begin{array}{l}\text { Salpingostomy, } \\
\text { donor oocytes }\end{array}$ & $\begin{array}{l}\text { Tubal } \\
\text { ectopic }\end{array}$ & NA & NA & $\begin{array}{l}2 \\
\text { Day } \\
3 \mathrm{ET}\end{array}$ & Frozen & NA & Abdominal $(H)$ & 8.5 weeks PT & No & $\begin{array}{l}\mathrm{KCl} \text { selective } \\
\text { reduction of } \\
\text { abdominal } \\
\text { pregnancy }\end{array}$ & $\begin{array}{l}\text { Selective } \\
\text { reduction of } \\
\text { abdominal } \\
\text { pregnancy, full } \\
\text { term viable IUP }\end{array}$ \\
\hline $\begin{array}{l}\text { Dmowski } \\
(2002) \\
{[34]}\end{array}$ & $\begin{array}{l}34 \text { yo } \\
\text { GOPO }\end{array}$ & Tubal & $\begin{array}{l}\text { Bilateral } \\
\text { Salpingectomy }\end{array}$ & No & $\begin{array}{l}\text { Long } \\
\text { protocol w/ } \\
\text { GnRH agonist }\end{array}$ & 15 & $\begin{array}{l}3 \\
\text { Day } \\
3 \mathrm{ET}\end{array}$ & Fresh & 38,635 & $\begin{array}{l}\text { Retroperitoneal } \\
\text { pancreatic }(E)\end{array}$ & 41 days PT & Yes & Laparotomy & $\begin{array}{l}\text { Retroperitoneal } \\
\text { subpancreatic } \\
\text { ectopic } \\
\text { removed by } \\
\text { laparotomy }\end{array}$ \\
\hline $\begin{array}{l}\text { Jain } \\
(2002) \\
\text { [35] }\end{array}$ & $\begin{array}{l}29 \text { yo } \\
\text { GOPO }\end{array}$ & Unexplained & NA & No & NA & NA & $\begin{array}{l}2 \\
\text { NA }\end{array}$ & NA & NA & $\begin{array}{l}\text { Pouch of } \\
\text { Douglas }(H)\end{array}$ & 9 weeks PT & NA & $\begin{array}{l}\text { Laparotomy at } \\
4 \text { w weeks (no } \\
\text { IUP seen), } \\
\text { selective } \\
\text { reduction of } \\
\text { ectopic at } \\
13 \text { weeks }\end{array}$ & $\begin{array}{l}\text { Selective } \\
\text { reduction of } \\
\text { abdominal } \\
\text { ectopic, } \\
\text { removal by } \\
\text { laparotomy, } \\
\text { SAB of IUP }\end{array}$ \\
\hline $\begin{array}{l}\text { Cormio } \\
\text { (2003) } \\
{[36]}\end{array}$ & $\begin{array}{l}30 \text { yo } \\
\text { G2P0020 }\end{array}$ & Tubal & $\begin{array}{l}\text { Bilateral } \\
\text { salpingectomy }\end{array}$ & $\begin{array}{l}\text { Tubal } \\
\text { ectopic x2 }\end{array}$ & $\begin{array}{l}\text { Menotropins, } \\
\text { hCG trigger }\end{array}$ & 7 & $\begin{array}{l}4 \\
\text { Day } \\
3 \mathrm{ET}\end{array}$ & Fresh & 256,400 & $\begin{array}{l}\text { Omentum, } \\
\text { uterine fundus } \\
\text { (H) }\end{array}$ & $\begin{array}{l}13 \text { weeks } \\
\text { gestation }\end{array}$ & No & Laparotomy & $\begin{array}{l}\text { Laparotomy } \\
\text { for abdominal } \\
\text { ectopic; Live } \\
\text { IUP delivered } \\
\text { at } 36 \text { weeks }\end{array}$ \\
\hline $\begin{array}{l}\text { Reid } \\
\text { (2003) } \\
\text { [37] }\end{array}$ & $\begin{array}{l}28 \text { yo } \\
\text { G5P1041 }\end{array}$ & Tubal & $\begin{array}{l}\text { bilateral } \\
\text { salpingectomy }\end{array}$ & $\begin{array}{l}\text { Tubal } \\
\text { ectopic x3 }\end{array}$ & NA & NA & $\begin{array}{l}3 \\
\text { NA }\end{array}$ & NA & 5500 & $\begin{array}{l}\text { Retroperitoneal, } \\
\text { iliac bifurcation } \\
\text { (E) }\end{array}$ & 63 days PT & NA & Laparotomy & $\begin{array}{l}\text { Removal of } \\
\text { ectopic via } \\
\text { laparotomy }\end{array}$ \\
\hline
\end{tabular}


Table 2 Abdominal ectopic case reports (Continued)

\begin{tabular}{|c|c|c|c|c|c|c|c|c|c|c|c|c|c|c|}
\hline $\begin{array}{l}\text { Kitade } \\
(2005) \\
{[38]}\end{array}$ & $\begin{array}{l}37 \text { yo } \\
\text { GOPO }\end{array}$ & Unexplained & NA & No & $\begin{array}{l}\text { Long } \\
\text { protocol w/ } \\
\text { GnRH agonist }\end{array}$ & 12 & $\begin{array}{l}3 \\
\text { Day } \\
3 \mathrm{ET}\end{array}$ & Fresh & 45,896 & $\begin{array}{l}\text { Splenic and } \\
\text { Tubal }(H)\end{array}$ & $\begin{array}{l}34 \text { days PT } \\
\text { (tubal), } 46 \text { day } \\
\text { PT (splenic) }\end{array}$ & $\begin{array}{l}\text { Tubal - } \\
\text { No, } \\
\text { Splenic - } \\
\text { Yes }\end{array}$ & $\begin{array}{l}\text { 1) Laparoscopic } \\
\text { salpingectomy } \\
\text { 2) Exploratory } \\
\text { laparotomy }\end{array}$ & $\begin{array}{l}\text { Removal of } \\
\text { tubal ectopic } \\
\text { by laparoscopy, } \\
\text { removal of } \\
\text { splenic ectopic } \\
\text { by laparotomy } \\
\text { (12 days later) }\end{array}$ \\
\hline $\begin{array}{l}\text { Ali } \\
\text { (2006) } \\
\text { [39] }\end{array}$ & $\begin{array}{l}35 \\
\text { NA }\end{array}$ & Tubal & Pelvic adhesions & No & NA & 11 & $\begin{array}{l}1 \\
\text { NA }\end{array}$ & Fresh & 1524 & $\begin{array}{l}\text { Tube with } \\
\text { Omental/ } \\
\text { peritoneal } \\
\text { trophoblastic } \\
\text { tissue }(H)\end{array}$ & $\begin{array}{l}3 \text { weeks PT - } \\
\text { tubal ectopic; } \\
5 \text { weeks PT - } \\
\text { omental tissue }\end{array}$ & No & $\begin{array}{l}\text { Laparoscopic } \\
\text { salpingectomy; } \\
\text { Laparocopic } \\
\text { removal of } \\
\text { omental/ } \\
\text { peritoneal } \\
\text { trophoblastic } \\
\text { tissue }\end{array}$ & $\begin{array}{l}\text { Removal of } \\
\text { tubal and } \\
\text { peritoneal// } \\
\text { omental } \\
\text { pregnancy } \\
\text { tissue by } 2 \\
\text { laparoscopies }\end{array}$ \\
\hline $\begin{array}{l}\text { Apantaku } \\
(2006) \\
{[40]}\end{array}$ & $\begin{array}{l}33 \\
\text { G3P1021 }\end{array}$ & Tubal & $\begin{array}{l}\text { Bilateral } \\
\text { salpingectomy }\end{array}$ & $\begin{array}{l}\text { Tubal } \\
\text { ectopic } \times 2\end{array}$ & NA & NA & $\begin{array}{l}2 \\
\text { NA }\end{array}$ & Fresh & NA & Right adnexa (E) & 6 weeks PT & No & Laparoscopy & $\begin{array}{l}\text { Laparoscopic } \\
\text { removal of } \\
\text { pregnancy } \\
\text { tissue }\end{array}$ \\
\hline $\begin{array}{l}\text { Knopman } \\
(2007) \\
{[41]}\end{array}$ & $\begin{array}{l}37 \text { yo } \\
\text { G4P0040 }\end{array}$ & Unexplained & NA & No & $\begin{array}{l}\text { GnRH } \\
\text { antagonist }\end{array}$ & 9 & $\begin{array}{l}2 \\
\text { Day } \\
5 \text { ET }\end{array}$ & Fresh & 1023 & $\begin{array}{l}\text { Posterior cul-de- } \\
\operatorname{sac}(H)\end{array}$ & $\begin{array}{l}7 \text { weeks, } \\
\text { nonviable IUP; } \\
9 \text { weeks } \\
\text { ectopic }\end{array}$ & Yes & Laparoscopy & $\begin{array}{l}\text { D\&C for non- } \\
\text { viable IUP; } \\
\text { Laparoscopy } \\
\text { for abdominal } \\
\text { ectopic }\end{array}$ \\
\hline $\begin{array}{l}\text { Shih } \\
\text { (2007) } \\
{[42]}\end{array}$ & $\begin{array}{l}33 \text { yo } \\
\text { GOPO }\end{array}$ & Male Factor & Patent tubes & No & $\begin{array}{l}\text { Long } \\
\text { protocol w/ } \\
\text { GnRH agonist }\end{array}$ & 4 & $\begin{array}{l}\text { NA } \\
\text { NA }\end{array}$ & Fresh & 901 & $\begin{array}{l}\text { Cul-de-sac } \\
\text { (E) }\end{array}$ & 28 days PT & No & $\begin{array}{l}\text { Laparoscopy } \\
\text { converted to } \\
\text { laparotomy }\end{array}$ & $\begin{array}{l}\text { Removal of } \\
\text { pregnancy } \\
\text { tissue by } \\
\text { laparotomy }\end{array}$ \\
\hline $\begin{array}{l}\text { Shojai } \\
(2007) \\
{[43]}\end{array}$ & $\begin{array}{l}35 \text { yo } \\
\text { GOPO }\end{array}$ & $\begin{array}{l}\text { Structural, DES } \\
\text { exposure }\end{array}$ & NA & No & NA & NA & $\begin{array}{l}3 \\
\text { NA }\end{array}$ & NA & NA & $\begin{array}{l}\text { Abdominal - } \\
\text { uterine fundus } \\
\text { (H) }\end{array}$ & $\begin{array}{l}21 \text { weeks } \\
\text { gestation }\end{array}$ & No & Laparotomy & $\begin{array}{l}\text { Delivery of } \\
\text { viable twins } \\
\text { at } 32 \text { weeks }\end{array}$ \\
\hline $\begin{array}{l}\text { Iwama } \\
(2008) \\
{[44]}\end{array}$ & $\begin{array}{l}31 \text { yo } \\
\text { G1P0010 }\end{array}$ & Tubal & $\begin{array}{l}\text { Right } \\
\text { Salpingectomy } \\
\text { for tubal ectopic } \\
\text { after IVF, left } \\
\text { salpingectomy } \\
\text { for hydrosalpinx }\end{array}$ & $\begin{array}{l}\text { Tubal } \\
\text { ectopic }\end{array}$ & NA & NA & $\begin{array}{l}3 \\
\text { Day } \\
3 \mathrm{ET}\end{array}$ & Fresh & 45,369 & $\begin{array}{l}\text { Inferior Vena } \\
\text { Cava/ } \\
\text { Retroperitoneal } \\
\text { (E) }\end{array}$ & $\begin{array}{l}32 \text { days PT: } \\
\text { PUL; } 53 \text { days } \\
\text { PT: } \\
\text { retroperitoneal } \\
\text { ectopic }\end{array}$ & Yes & $\begin{array}{l}\text { D\&C, MTX, } \\
\text { Diagnostic } \\
\text { laparoscopy, } \\
\text { repeat MTX, } \\
\text { Exploratory } \\
\text { laparotomy }\end{array}$ & $\begin{array}{l}\text { Ruptured } \\
\text { retroperitoneal } \\
\text { ectopic, } \\
\text { removed by } \\
\text { laparotomy }\end{array}$ \\
\hline $\begin{array}{l}\text { Hyvarinen } \\
\text { (2009) [45] }\end{array}$ & $\begin{array}{l}\text { NA } \\
\text { NA }\end{array}$ & NA & NA & NA & NA & NA & $\begin{array}{l}\text { NA } \\
\text { NA }\end{array}$ & NA & NA & Abdominal (E) & $\begin{array}{l}30 \text { weeks } \\
\text { gestation }\end{array}$ & No & Laparotomy & $\begin{array}{l}\text { Delivery of } \\
\text { viable fetus } \\
\text { at } 30 \text { weeks }\end{array}$ \\
\hline $\begin{array}{l}\text { Zacche } \\
\text { (2011) } \\
{[46]}\end{array}$ & $\begin{array}{l}36 \\
\text { G1P1 }\end{array}$ & Tubal & $\begin{array}{l}\text { Bilateral } \\
\text { Salpingectomy, } \\
\text { PID }\end{array}$ & No & NA & NA & $\begin{array}{l}2 \\
\text { NA }\end{array}$ & Fresh & NA & Abdominal $(H)$ & $\begin{array}{l}32 \text { weeks at } \\
\text { Cesarean } \\
\text { Delivery }\end{array}$ & No & $\begin{array}{l}\text { Laparotomy, } \\
\text { hysterectomy }\end{array}$ & $\begin{array}{l}\text { Viable twin } \\
\text { pregnancies } \\
\text { at } 32 \text { weeks; } \\
\text { Hysterectomy }\end{array}$ \\
\hline
\end{tabular}


Table 2 Abdominal ectopic case reports (Continued)

\begin{tabular}{|c|c|c|c|c|c|c|c|c|c|c|c|c|c|c|}
\hline $\begin{array}{l}\text { Angelova } \\
(2015) \\
{[47]}\end{array}$ & $\begin{array}{l}33 \\
\text { NA }\end{array}$ & Male Factor & $\begin{array}{l}\text { Obturated } \\
\text { left tube }\end{array}$ & NA & $\begin{array}{l}\text { Short } \\
\text { protocol, w/ } \\
\text { GnRH } \\
\text { antagonist }\end{array}$ & NA & $\begin{array}{l}2 \\
\text { Day } \\
3 \mathrm{ET}\end{array}$ & Fresh & NA & $\begin{array}{l}\text { Abdominal - } \\
\text { vesicouterine } \\
\text { junction (E) }\end{array}$ & 23 days PT & No & Laparoscopy & $\begin{array}{l}\text { Laparoscopic } \\
\text { removal of } \\
\text { pregnancy } \\
\text { tissue }\end{array}$ \\
\hline $\begin{array}{l}\text { Dalmia } \\
(2015) \\
{[48]}\end{array}$ & $\begin{array}{l}37 \\
\text { G1P0010 }\end{array}$ & $\begin{array}{l}\text { EndometriosisTubal } \\
\text { factor }\end{array}$ & $\begin{array}{l}\text { Bilateral } \\
\text { salpingectomy } \\
\text { for hydrosalpinx }\end{array}$ & NA & NA & NA & $\begin{array}{l}\text { NA } \\
\text { NA }\end{array}$ & NA & 21,730 & Left adnexa (E) & 2 weeks PT & No & $\begin{array}{l}\text { Mini- } \\
\text { laparotomy }\end{array}$ & $\begin{array}{l}\text { Removal of } \\
\text { ectopic via } \\
\text { laparotomy }\end{array}$ \\
\hline $\begin{array}{l}\text { Koyama } \\
(2015) \\
{[49]}\end{array}$ & $\begin{array}{l}32 \\
\text { G5P1 }\end{array}$ & Male Factor & NA & No & NA & NA & $\stackrel{1}{\mathrm{NA}}$ & Frozen & 14,800 & $\begin{array}{l}\text { Retroperitoneal } \\
\text { (E) }\end{array}$ & $\begin{array}{l}10 \text { weeks } \\
\text { gestation }\end{array}$ & NA & Laparoscopy & $\begin{array}{l}\text { Laparoscopic } \\
\text { removal of } \\
\text { pregnancy } \\
\text { tissue }\end{array}$ \\
\hline
\end{tabular}

Abbreviations: $A B$ Abortion, $D \& C$ Dilation and curettage, DES Diethylstilbestrol, $E$ Ectopic, FSH Follicle stimulating hormone, GnRH Gonadotropin-releasing hormone, $H$ Heterotopic, $h C G$ Human chorionic gonadotropin, hMG Human menopausal gonadotropin, HSG Hysterosalpingogram, IUP Intrauterine pregnancy, IVF In vitro fertilization, KCI Potassium chloride, MTX Methotrexate, NA Not available, PID Pelvic inflammatory disease, PT Post transfer, RBC Red blood cell, TC Technetium, SAB Spontaneous abortion 
review demonstrates several trends among reported cases. First, the majority of cases $(61 \%)$ report a history of anatomic/structural infertility etiology with history of tubal factor infertility (TFI) (46\%) being the most common. This is consistent with TFI being a known risk factor for ectopic pregnancy following IVF. One study that examined the risk factors for EP following IVF in 712 women reported an odds ratio (OR) of 3.99 (95\% CI: 1.23 to 12.98) for women with TFI compared to those with other infertility causes [12]. In a larger, more recent study of 553,577 ART cycles in the US, among all infertility diagnoses, TFI was the only one significantly associated with increased risk for ectopic pregnancy (adjusted relative risk (RR) 1.25, 95 \% CI 1.16-1.35) [13]. In addition, history of tubal ectopic pregnancy was particularly common, being reported in $37 \%$ of the abdominal ectopic cases. This also appears to be consistent with the general ART-associated EP literature. A retrospective study that measured the risk of EP following IVF in 181 women with a previous ectopic demonstrated a 45-fold higher risk of recurrence when compared with 377 women with other causes of infertility. The authors reported that the prevalence of EP was $8.95 \%$ compared with $0.75 \%$ in the control group [14]. History of prior tubal surgery was also particularly common (50 \%) among abdominal ectopic cases in our systematic review. A history of tubal/pelvic surgery is another major risk factor for the development of EP following IVF. Odds ratio for developing EP was 8.52 (95 \% CI: 5.91-12.27) for prior adnexal surgery, 11.02 (95 \% CI: 5.49-22.15) for a previous tubal infertility surgery, 5.16 (95 \% CI: 1.25-21.21) for prior surgery for endometriosis and 17.70 (95 \% CI: 8.11-38.66) for a previous abdominal/ pelvic surgery $[12,15,16]$. Interestingly, bilateral salpingectomy was the most common tubal surgery reported in our case review. While the exact mechanism of abdominal ectopic after bilateral salpingectomy remains unclear, many authors have proposed that it may be due to the development of a micro-fistulous tract after salpingectomy. Uterine perforation during embryo transfer has also been suggested as a mechanism for abdominal ectopic pregnancy, and embryo transfer technique has been related to overall EP risk after IVF. Aspects of the transfer that may increase risk of EP include large volume of transfer media, induction of abnormal uterine contractions, and location of embryo transfer in relation to the uterine fundus [9]. These factors have all been associated with retrograde flow of both transfer media and the embryo toward the fallopian tubes. Many suggestions have been made regarding optimal transfer location within the endometrium, ranging from 5 to $20 \mathrm{~mm}$ from the fundal surface, while others recommend "mid-cavity" location to avoid proximity to the fallopian tubes [17-19].
Other trends identified in our systematic review include $>1$ embryo transferred (reported in $79 \%$ of cases) and a large number of heterotopic abdominal pregnancy (reported in $46 \%$ of cases). Multiple embryo transfer has always been associated with increased risk of EP with transfer of two or less embryos carrying lower risk than after three or more embryos [20]. In the setting of multiple embryo transfers, identification of an intrauterine pregnancy often leads to delayed diagnosis of abdominal pregnancy in the absence of clinical symptoms. Among the heterotopic cases, 4 reported a 2 week delay in diagnosis of the abdominal ectopic from the time of suspected ectopic, and 5 cases did not identify the abdominal ectopic until beyond the 12th week of pregnancy. Unfortunately, this type of delayed diagnosis has the potential to lead to significantly morbid outcomes. In our review, four cases of viable abdominal pregnancies were identified, which is an extremely rare outcome. Three of these cases were identified at 19 weeks or beyond, and all three had attachment of the abdominal placenta to the peritoneal surface of the uterus without involvement of other abdominal organs. Placental attachment to the uterus has previously been associated with viability of abdominal pregnancies [21], and with a relatively lower risk of bleeding and lower likelihood of fetal growth retardation [22].

Finally, abdominal ectopic pregnancies were far more common in fresh embryo transfer (71\% of cases) than frozen embryo transfer (11\% of cases). This may be due to the fact that frozen embryo transfer has become widely used only recently, and we may begin to see higher frequency with frozen embryo transfers over time. However, several recent studies indicate that ectopic pregnancy rates are higher for fresh as compared to frozen IVF cycles $[1,6]$.

A limitation of this review is the heterogeneity of reported cases and IVF practices which encompass several decades. Further research focusing on more homogenous population may help in better characterizing this rare IVF complication.

\section{Conclusions}

In conclusion, ectopic pregnancy, including abdominal ectopic, is a known risk of IVF. The case reported highlights the diagnostic challenges behind this rare form of ectopic pregnancy, and the need to keep it in the differential in atypical ectopic presentations. Our systematic literature review has revealed several trends in reported cases of abdominal ectopic pregnancy after IVF including tubal factor infertility, history of tubal ectopic and tubal surgery, higher number of embryos transferred, and fresh embryo transfers. These are consistent with known risk factors for ectopic pregnancy following IVF. 


\section{Abbreviations}

AB: Abortion; ART: Assisted reproduction technologies; D\&C: Dilation and curettage; DES: Diethylstilbestrol; E: Ectopic; FSH: Follicle stimulating hormone; GnRH: Gonadotropin-releasing hormone; H: Heterotopic; hCG: Human chorionic gonadotropin; hMG: Human menopausal gonadotropin; HSG: Hysterosalpingogram; IUP: Intrauterine pregnancy; IVF: In vitro fertilization; KCl: Potassium chloride; MTX: Methotrexate; NA: Not available; PID: Pelvic inflammatory disease; PT: Post transfer; RBC: Red blood cell; SAB: Spontaneous abortion; Tc: Technetium

\section{Acknowledgements}

None.

\section{Funding}

None.

\section{Availability of data and materials}

Not applicable.

\section{Authors' contributions}

NY performed the systematic literature search, extracted and analyzed the data, and wrote the manuscript; RT conceived and designed the study, critically reviewed and revised the manuscript; JRM conceived the study, critically reviewed and revised the manuscript. All authors read and approved the final submission.

\section{Competing interests}

The authors declare that they have no competing interests.

\section{Consent for publication}

Not applicable.

\section{Ethics approval and consent to participate}

Since this study used only deidentified patient data, and published data from the literature, no approval from our institutional review board (IRB) was required.

\section{Received: 31 August 2016 Accepted: 6 October 2016}

Published online: 19 October 2016

\section{References}

1. Londra L, Moreau C, Strobino D, Garcia J, Zacur H, Zhao Y. Ectopic pregnancy after in vitro fertilization: differences between fresh and frozenthawed cycles. Fertil Steril. 2015;104(1):110-8

2. Clayton $H B$, Schieve LA, Peterson HB, Jamieson DJ, Reynolds MA, Wright VC Ectopic pregnancy risk with assisted reproductive technology procedures. Obstet Gynecol. 2006;107(3):595-604.

3. Bouyer J, Coste J, Fernandez H, Pouly JL, Job-Spira N. Sites of ectopic pregnancy: a 10 year population-based study of 1800 cases. Hum Reprod. 2002;17(12):3224-30.

4. Alto WA. Abdominal pregnancy. Am Fam Physician. 1990;41(1):209-14

5. Zhang Y-L, Sun J, Su Y-C, Guo Y-H, Sun Y-P. Study on the incidence and influences on ectopic pregnancy from embryo transfer of fresh cycles and frozen-thawed cycles. Zhonghua Fu Chan Ke Za Zhi. 2012:47(9):655-8.

6. Huang B, Hu D, Qian K, et al. Is frozen embryo transfer cycle associated with a significantly lower incidence of ectopic pregnancy? An analysis of more than 30,000 cycles. Fertil Steril. 2014;102(5):1345-9.

7. Decleer W, Osmanagaoglu K, Meganck G, Devroey P. Slightly lower incidence of ectopic pregnancies in frozen embryo transfer cycles versus fresh in vitro fertilization-embryo transfer cycles: a retrospective cohort study. Fertil Steril. 2014;101(1):162-5.

8. Rombauts L, McMaster R, Motteram C, Fernando S. Risk of ectopic pregnancy is linked to endometrial thickness in a retrospective cohort study of 8120 assisted reproduction technology cycles. Hum Reprod. 2015. doi:10. 1093/humrep/dev249.

9. Refaat B, Dalton E, Ledger WL. Ectopic pregnancy secondary to in vitro fertilisation-embryo transfer: pathogenic mechanisms and management strategies. Reprod Biol Endocrinol. 2015;13:30.

10. Atrash HK, Friede A, Hogue CJ. Abdominal pregnancy in the United States: frequency and maternal mortality. Obstet Gynecol. 1987;69(3 Pt 1):333-7.
11. Fisch B, Peled Y, Kaplan B, Zehavi S, Neri A. Abdominal pregnancy following in vitro fertilization in a patient with previous bilateral salpingectomy. Obstet Gynecol. 1996;88(4 Pt 2):642-3.

12. Malak M, Tawfeeq T, Holzer H, Tulandi T. Risk factors for ectopic pregnancy after in vitro fertilization treatment. J Obstet Gynaecol Can. 2011;33(6):617-9.

13. Perkins KM, Boulet SL, Kissin DM, Jamieson DJ, National ART Surveillance (NASS) Group. Risk of ectopic pregnancy associated with assisted reproductive technology in the United States, 2001-2011. Obstet Gynecol. 2015;125(1):70-8.

14. Weigert M, Gruber D, Pernicka E, Bauer P, Feichtinger W. Previous tubal ectopic pregnancy raises the incidence of repeated ectopic pregnancies in in vitro fertilization-embryo transfer patients. J Assist Reprod Genet. 2009; 26(1):13-7.

15. Parashi S, Moukhah S, Ashrafi M. Main risk factors for ectopic pregnancy: a case-control study in a sample of Iranian women. Int J Fertil Steril. 2014;8(2):147-54.

16. Li C, Meng C-X, Zhao W-H, Lu H-Q, Shi W, Zhang J. Risk factors for ectopic pregnancy in women with planned pregnancy: a case-control study. Eur J Obstet Gynecol Reprod Biol. 2014;181:176-82

17. Rovei $V$, et al. IVF outcome is optimized when embryos are replaced between 5 and $15 \mathrm{~mm}$ from the fundal endometrial surface: a prospective analysis on 1184 IVF cycles. Reprod Biol Endocrinol. 2013;11:114

18. Nazari A, Askari HA, Check JH, O'Shaughnessy A. Embryo transfer technique as a cause of ectopic pregnancy in in vitro fertilization. Fertil Steril. 1993;60:919-21.

19. Coroleu B, et al. The influence of the depth of embryo replacement into the uterine cavity on implantation rates after IVF: a controlled, ultrasound guided study. Hum Reprod. 2002;17:341-6.

20. Pandian Z, Marjoribanks J, Ozturk O, Serour G, Bhattacharya S. Number of embryos for transfer following in vitro fertilisation or intra-cytoplasmic sperm injection. Cochrane Database Syst Rev. 2013;7:CD003416.

21. Dubinsky TJ, Guerra F, Gormaz G, Maklad N. Fetal survival in abdominal pregnancy: a review of 11 cases. J Clin Ultrasound. 1996;24:513-7.

22. Huang K, Song L, Wang L, Gao Z, Meng Y, Lu Y. Advanced abdominal pregnancy: an increasingly challenging clinical concern for obstetricians. Int J Clin Exp Pathol. 2014;7(9):5461-72.

23. Oehninger S, Kreiner D, Bass MJ, Rosenwaks Z. Abdominal pregnancy after in vitro fertilization and embryo transfer. Obstet Gynecol. 1988;72(3 Pt 2):499-502.

24. Bassil S, Pouly JL, Canis M, et al. Advanced heterotopic pregnancy after invitro fertilization and embryo transfer, with survival of both the babies and the mother. Hum Reprod. 1991;6(7):1008-10.

25. Ferland RJ, Chadwick DA, O'Brien JA, Granai 3rd CO. An ectopic pregnancy in the upper retroperitoneum following in vitro fertilization and embryo transfer. Obstet Gynecol. 1991;78(3 Pt 2):544-6.

26. Ragni G, Lombroso Finzi GC, Olivares MD, Crosignani PG. Twin in vitro fertilization (IVF) pregnancies: spontaneous intrauterine abortion after selective second-trimester termination of ectopic intraabdominal pregnancy. J In Vitro Fert Embryo Transf. 1991;8(4):236-7.

27. Balmaceda JP, Bernardini L, Asch RH, Stone SC. Early primary abdominal pregnancy after in vitro fertilization and embryo transfer. J Assist Reprod Genet. 1993;10(4):317-20.

28. Fisch B, Powsner $E$, Heller $L$, et al. Heterotopic abdominal pregnancy following in-vitro fertilization/embryo transfer presenting as massive lower gastrointestinal bleeding. Hum Reprod. 1995;10(3):681-2.

29. delRosario R, el-Roeiy A. Abdominal pregnancy on the bladder wall following embryo transfer with cryopreserved-thawed embryos: a case report. Fertil Steril. 1996;66(5):839-41.

30. Moonen-Delarue MW, Haest JW. Ectopic pregnancy three times in line of which two advanced abdominal pregnancies. Eur J Obstet Gynecol Reprod Biol. 1996;66(1):87-8

31. Pisarska MD, Casson PR, Moise Jr KJ, DiMaio DJ, Buster JE, Carson SA. Heterotopic abdominal pregnancy treated at laparoscopy. Fertil Steril. 1998;70(1):159-60.

32. Deshpande N, Mathers A, Acharya U. Broad ligament twin pregnancy following in-vitro fertilization. Hum Reprod. 1999;14(3):852-4.

33. Scheiber MD, Cedars MI. Case Report: Successful non-surgical management of a heterotopic abdominal pregnancy following embryo transfer with cryopreserved-thawed embryos. Hum Reprod. 1999;14(5):1375-7.

34. Dmowski WP, Rana N, Ding J, Wu WT. Retroperitoneal subpancreatic ectopic pregnancy following in vitro fertilization in a patient with previous 
bilateral salpingectomy: how did it get there? J Assist Reprod Genet. 2002;19(2):90-3.

35. Jain S, Justus K, Bober S. Selective transvaginal embryo reduction in heterotopic pregnancy located intra-abdominally. J Obstet Gynaecol. 2002;22(3):330.

36. Cormio G, Santamato S, Putignano G, Bettocchi S, Pascazio F. Concomitant abdominal and intrauterine pregnancy after in vitro fertilization in a woman with bilateral salpingectomy. A case report. J Reprod Med. 2003;48(9):747-9.

37. Reid F, Steel M. An exceptionally rare ectopic pregnancy. BJOG. 2003; 110(2):222-3.

38. Kitade M, Takeuchi H, Kikuchi I, Shimanuki H, Kumakiri J, Kinoshita K. A case of simultaneous tubal-splenic pregnancy after assisted reproductive technology. Fertil Steril. 2005;83(4):1042.

39. Ali CR, Fitzgerald C. Omental and peritoneal secondary trophoblastic implantation - an unusual complication after IVF. Reprod Biomed Online. 2006;12(6):776-8.

40. Apantaku O, Rana P, Inglis T. Broad ligament ectopic pregnancy following in-vitro fertilisation in a patient with previous bilateral salpingectomy. J Obstet Gynaecol. 2006;26(5):474.

41. Knopman JM, Talebian S, Keegan DA, Grifo JA. Heterotopic abdominal pregnancy following two-blastocyst embryo transfer. Fertil Steril. 2007;88(5): 1437. e13-e15.

42. Shih C-C, Lee RK-K, Hwu Y-M. Cul-de-sac pregnancy following in vitro fertilization and embryo transfer. Taiwan J Obstet Gynecol. 2007;46(2):171-3.

43. Shojai R, Chaumoitre K, Chau C, Panuel M, Boubli L, d'Ercole C. Advanced combined abdominal and intrauterine pregnancy: a case report. Fetal Diagn Ther. 2007;22(2):128-30.

44. Iwama H, Tsutsumi S, Igarashi H, Takahashi K, Nakahara K, Kurachi H. A case of retroperitoneal ectopic pregnancy following IVF-ET in a patient with previous bilateral salpingectomy. Am J Perinatol. 2008;25(1):33-6.

45. Hyvärinen M, Raudaskoski T, Tekay A, Herva R. Abdominal pregnancy. Duodecim. 2009;125(22):2448-51.

46. Zacchè MM, Zacchè G, Gaetti L, Vignali M, Busacca M. Combined intrauterine and abdominal pregnancy following ICSI with delivery of two healthy viable fetuses: a case report. Eur J Obstet Gynecol Reprod Biol. 2011; 154(2):232-3.

47. Angelova MA, Kovachev EG, Kozovski I, Kornovski YD, Kisyov SV, Ivanova VR. A case of secondary abdominal pregnancy after In Vitro Fertilization PreEmbryo Transfer (IVF-ET). Open Access Maced J Med Sci. 2015;3(3):426-8.

48. Dalmia R, Murthy J, Orakwue C. A case of abdominal pregnancy following in vitro fertilization in a patient with previous bilateral salpingectomy. IJMPCR. 2015;2(1):18-21.

49. Koyama S, Yoshino A, Okuno K, et al. A case of abdominal pregnancy following in vitro fertilization and embryo transfer treated with laparoscopic surgery. Gynecol Minim Invasive Ther. doi:10.1016/j.gmit.2015.04.006.

\section{Submit your next manuscript to BioMed Central and we will help you at every step:}

- We accept pre-submission inquiries

- Our selector tool helps you to find the most relevant journal

- We provide round the clock customer support

- Convenient online submission

- Thorough peer review

- Inclusion in PubMed and all major indexing services

- Maximum visibility for your research

Submit your manuscript at www.biomedcentral.com/submit

CBiomed Central 